\title{
Acute care hospital at different levels of intensity: the role of Geriatrician
}

\author{
Ilaria Liguori ${ }^{1}$ - Gennaro Russo ${ }^{1}$ - Luisa Aran ${ }^{1}$. Giulia Bulli ${ }^{1}$. Francesco Curcio ${ }^{1}$. David Della-Morte ${ }^{2,3}$. \\ Gaetano Gargiulo $^{4} \cdot$ Gianluca Testa $^{1,5} \cdot$ Andrea Ungar $^{6} \cdot$ Francesco Cacciatore $^{1,7} \cdot$ Domenico Bonaduce $^{1}$. \\ Pasquale Abete ${ }^{1}$
}

Received: 3 November 2017 / Accepted: 15 February 2018 / Published online: 21 February 2018

(c) Springer International Publishing AG, part of Springer Nature 2018

\begin{abstract}
The traditional model of care is based on "disease-centered" management that requires the organization of the hospital in specialized wards, to which the patient is assigned for the main disease. The growing need to optimize economical and human resources and to promote a global approach to the patient has led to the setting up of the intensity of care model. It is a health system based on a "patient-centered" approach, where the hospital is organized in departments dedicated to patients with homogenous needs of care. In Italy, intensity of care model is currently being tested in the hospital organization, where three levels of intensity are proposed: low, medium and high. The purpose of the following review is to describe the role and importance of the Geriatrician in each of these care settings and to highlight the contradiction of a National Health System which promotes the geriatric approach to all types of patients, but does not invest in the formation and integration of the figure of the Geriatrician in clinical practice, condemning it to marginalization or even extinction.
\end{abstract}

Keywords Acute care hospital $\cdot$ Level of care intensity $\cdot$ Geriatrician

\section{Introduction}

The contemporary medicine has often considered "disease" as the centre of the care process. So, the traditional model of clinical practice is "disease-centred" and characterized by a reductionist division of body in its basic components. In

Pasquale Abete

p.abete@unina.it

1 Department of Translational Medical Sciences, University of Naples "Federico II", Via S. Pansini, 80131 Naples, Italy

2 Department of Systems Medicine, University of Rome Tor Vergata, Rome, Italy

3 San Raffaele Roma Open University, 00166 Rome, Italy

4 Division of Internal Medicine, AOU San Giovanni di Dio e Ruggi di Aragona, Salerno, Italy

5 Department of Medicine and Health Sciences, University of Molise, Campobasso, Italy

6 Azienda Ospedaliero-Universitaria Careggi, University of Florence, Florence, Italy

7 Heart Transplantation Unit, Azienda Ospedaliera dei Colli, Monaldi Hospital, Naples, Italy this model, the patient is considered only for his biological features and "disease" represents an organic lesion which can be objectified and measured using physical and chemical parameters. Thus, the patient becomes a passive subject because he only receives care by healthcare professionals and the environment remains irrelevant in the healthcare process [1]. As regards Italian hospital organization, traditional healthcare model provides that patients with acute conditions access to emergency department where they are assigned a colour code corresponding to the severity of their clinical condition through the triage. Subsequently, patients are managed in a short-stay observation unit (SOU) or an emergency medicine division. When they have been stabilized or when a diagnosis is formulated, patients are addressed to the reference specialist ward. Therefore, hospital is organized in specialist wards (i.e. nephrology, cardiology, pneumology) and the patient is sent to one of them according to the prevalent disease and not for the individual needs of the patient.

On the contrary, "patient-centred" medicine is an healthcare model which focus medical attention on patient's health needs and outcomes, rather than the doctor's [2], according to the bio-psycho-social medicine model proposed by 
Engel [3]. Based on the assumption that personal narratives, emotions and subjective perceptions of the patients must be taken into account by healthcare professionals, we have to integrate the concept of "curing" with "caring". In this scenario, the concept of "disease", intended as a group of signs and symptoms, should be integrated with "illness", which includes personal emotions, desires, expectations and social context, and "sickness", as a social expression of unhealthy [1].

In this perspective, the hospital should be no longer subdivided into departments, but in areas/sectors dedicated to patients with homogeneous needs of care. In this way, it is possible to optimize the use of economic and human resources, to reduce costs and to provide higher quality performances [4]. Thus, to each level of intensity of care corresponds a different grade of complexity, on which are based the needs of the healthcare professionals. For example, nursing requirements are expressed through nursing/hospital patient ratio in relation to the gravity. In this model, the care process provides a multidisciplinary approach which involves different specialists, healthcare professionals and nursing staff, but also relatives and caregivers, to provide a patient-tailored assistance [5].

\section{The role of Geriatrician in emergency department: the "Silver Code"}

The number of visits to emergency department (ED) has significantly increased during the past few years, especially for elderly patients (26\% more from 1993 to 2003) [6]. The complex management of acute conditions in the elderly derives from their frailty, which is characterized by comorbidity, polypharmacy, disability, and lack of an adequate social support network [7, 8]. Frail older patients are also at high risk for hospital readmissions and adverse health outcomes, such as functional or cognitive decline, adverse drug reactions, or death $[9,10]$. Furthermore, another element of complexity is the atypical clinical presentation. In fact, a recent study has showed that on 633 older adults who visit the ED the prevalence of atypical presentation is $28.6 \%$ (181 of 633 cases). The three main unusual presentations of diseases are the failure to develop fever with a disease known to cause fever (i.e. tuberculosis, infective endocarditis, lung abscess, pneumonia, urinary tract infection, septic shock, systemic infection, skin and soft tissue infection and acute abdominal condition, e.g. appendicitis), followed by the lack of pain with a disease known to cause pain (i.e. acute coronary syndrome, obstructive uropathy, and acute abdominal condition, e.g. acute appendicitis, liver abscess) and fatigue [11]. In particular, an observational study shows that a higher complexity of acute illness is associated to a more severe grade of frailty and both are independently related to inpatient mortality [12]. Because of these characteristics of frailty, it has been hypothesized that high risk ED older patients could benefit more from admission to acute geriatric unit care than to internal medicine wards. To test this hypothesis was developed and validated the "Silver Code", a prognostic tool to identify older patients at an increased risk for 1-year mortality regardless the reason for the index hospitalization, probably reflecting patients baseline health conditions. In addition, the same study evaluated potential differences in mortality rates among patients transferred to an acute geriatric unit care compared to those transferred to internal medicine wards. The results of this study showed that higher risk of mortality, characterized by higher scores of the "Silver Code" $(\geq 7)$, were associated with better outcomes when elderly patients were assigned to acute geriatric unit than to internal medicine wards (Fig. 1) [13]. These results underline the importance of geriatric care in the management of elderly patients also during acute conditions. However, the distribution of acute geriatric beds in Regione Campania is alarming: for 1,045,084 people older than 65 years less than 100 acute geriatric beds (Abete, unpublished data).

\section{The role of Geriatrician in hospital by intensity of care}

In the US, hospital organization based on intensity of care includes two models: the "Progressive Patient Care", for all patients, and the "Chronic Care Model", specific for chronic disease. In the first model, patients are divided accordingly to their complexity in four care units: intensive care, intermediated care, minimal care and organized home care. Instead, in Italy, there is no established tradition about hospital organization based on intensity of care [14].

Actually, in Italy, pilot experiments on a reorganization of hospitals are underway in different Regions, among which Regione Toscana, Piemonte, Lombardia and Emilia Romagna. To name just a few, the Local Healthcare Company (LHC) 2 in Lucca in Tuscany, followed by other examples in Tuscany [15]; the hospital of the City of Health and Science of Turin [16]; the S. Gerardo Hospital in Monza [17]; the Hospice Company of Vimercate [18]; the Civil Hospital of Legnano [19]; the Galliera of Genoa [20]; the LHC 3 in Umbria [21].

Therefore, there is not an "Italian model" for hospital organization by intensity of care. Nevertheless, in existing experiences, it is possible to identify three levels of care where patients are allocated homogeneously according to their needs [22, 23]:

- Level 1-high intensity of care: includes intensive therapy and sub-intensive therapy; in the hospital, the 

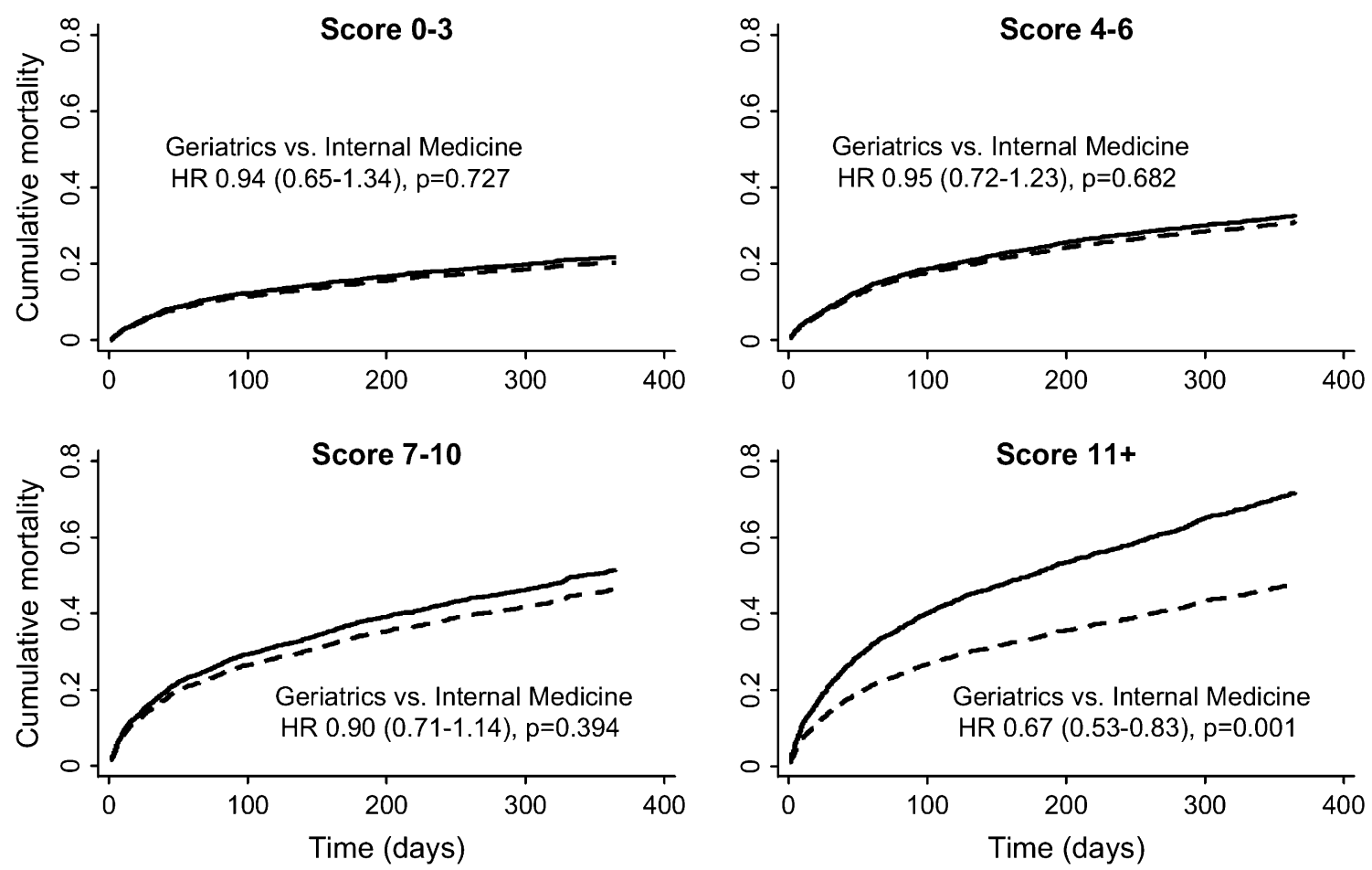

Internal Medicine $\quad-\quad$ Geriatrics

Fig. 1 1-year survival as a function of hospital admission to an acute geriatrics or internal medicine ward, after stratification by risk of death score. Cox regression models, adjusted for age, sex, marital

patient in danger of life or critical condition is under close medical supervision to avoid complications or acute organ failures. It corresponds to the intensive care of the US "Progressive Patient Care".

- Level 2-medium intensity of care: organized in functional areas, it includes ordinary and short-term hospitalization; presupposes that patients stay at least one night in the hospital (i.e. week surgery, 1-day surgery); the goal is the clinical state stabilization and the monitoring of vital parameters of patient without continuous observation. It corresponds to the intermediate care of the US "Progressive Patient Care".

- Level 3-low intensity of care: dedicated to post-acute care, it is similar to the sub-acute care; the patient is provided with unsupervised assistance at the final stage of his way to the territorial services. It corresponds to the minimal and organized home care of the US "Progressive Patient Care" [24].

Now we are going to present some clinical cases to clarify the role of Geriatrician in these settings.

Figure 2 shows the transition from traditional to intensity of care model. status, previous admission to a day hospital, previous admission to a regular ward and the corresponding discharge diagnosis, and number of drugs taken. (with permission [13])

\section{High intensity scenario}

A 83-years-old male accesses to ED in critical conditions, presenting severe dyspnoea, diaphoresis, paleness, confusion. His symptoms, physical examination, blood and imaging exams are showed in Table 1. His medical history includes post-ischemic dilative cardiomyopathy complicated by mitral insufficiency and permanent atrial fibrillation; chronic obstructive pulmonary disease (COPD), colon diverticulosis, and previous transient ischemic attack (TIA). The following diagnosis is formulated: acute cardiorespiratory failure for pulmonary oedema and respiratory exacerbation in patient with chronic obstructive pulmonary disease and post-ischemic dilative cardiomyopathy complicated by atrial fibrillation with high ventricular response. The therapy was performed as described in Table 1. After few hours, the patient, despite an initial improvement of his clinical conditions, shows psychomotor agitation. The consulted Geriatrician diagnoses delirium and recommends replacing ceftazidime with meropenem, initiating low-dose haloperidol and rapid nesting from non-invasive ventilator with transfer to a lower intensity division of care. 
Fig. 2 Transition from classical colour code to care intensity code (see text for the explanation). $E D$ emergency department, $S O U$ short-stay observation unit

\section{Traditional Model (Specialist medicine)}

Current Model (Intensity of care)

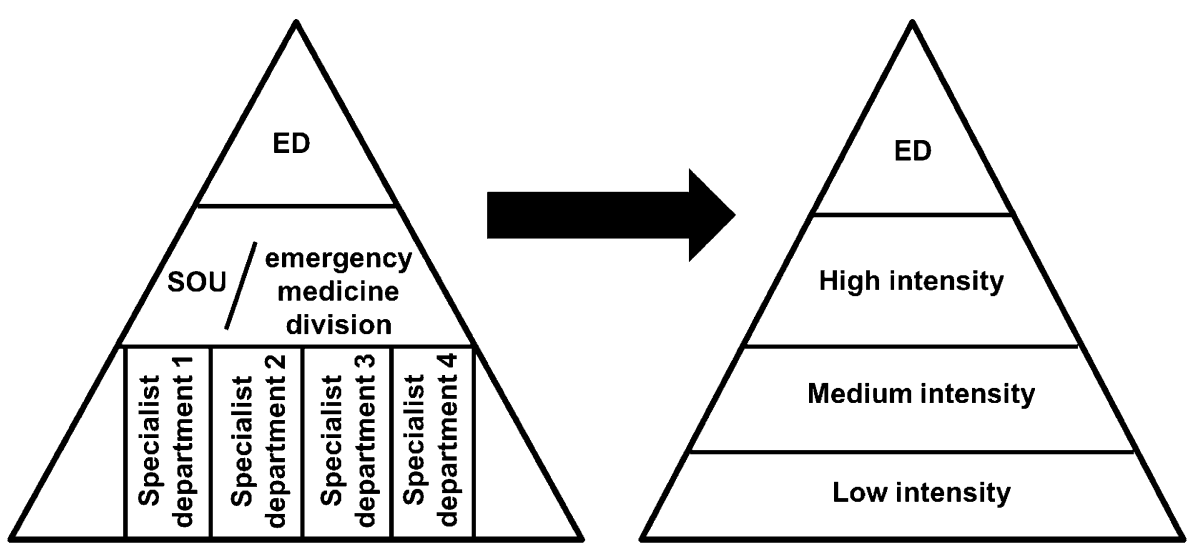

Table 1 High intensity scenario

Male, 83 years old

Symptoms

Vital signs

Thoracic examination

ECG

Chest X-ray

Blood gas analysis

Laboratory tests

Therapy
Severe dyspnea, diaphoresis, paleness, confusion

BP $180 / 100 \mathrm{mmHg}$

HR 150 beats/min

RR 30 breaths/min

Gasps as rising tide

Atrial fibrillation

Bilateral mid-basal pleural effusion with signs of apical interstitial infiltrate

$\mathrm{SpO}_{2} 82 \%, \mathrm{pH} 7.34, \mathrm{pO}_{2} 50 \mathrm{mmHg}, \mathrm{pCO}_{2} 50 \mathrm{mmHg}$ in Venturi mask at an $\mathrm{FiO}_{2} 50 \%$

$\mathrm{Hb} 11.30 \mathrm{~g} \%$; WBC $12.500 \mathrm{~mm}^{3}$ (neutrophils $85.5 \%$ ), fibrinogen $652 \mathrm{mg} \%$, glycemia $320 \mathrm{mg} \%$, INR 3

Furosemide $100 \mathrm{mg}$ e.v, nitro-glycerine $5 \mathrm{mg} 4 \mathrm{fl}$ in $\mathrm{NaCl} 0.9 \% 500 \mathrm{cc}$ e.v. $30 \mathrm{ml} / \mathrm{h}$, amiodarone $150 \mathrm{mg} 8 \mathrm{fl}$ in glucose solution $5 \% 500 \mathrm{cc}$ e.v. $30 \mathrm{ml} / \mathrm{h}$, ceftazidime $1 \mathrm{gr} \times 3$ e.v., levofloxacin $500 \mathrm{mg}$ e.v., enoxaparin $6000 \mathrm{UI} f \mathrm{fl} 2$ s.c. and non-invasive ventilation (NIV)

$B P$ blood pressure, $H R$ heart rate, $b p m$ beats per minute, $R R$ respiratory rate, $E C G$ electrocardiogram, $\mathrm{FiO}_{2}$ fraction of inspired oxygen, $\mathrm{Hb}$ haemoglobin, WBC white blood cells, INR international normalized ratio

Atypical presentation is very common in elderly patients [11]. In particular, data from the "Osservatorio Geriatrico Campano" show a reduced incidence of dyspnoea as clinical presentation in elderly patients with chronic heart failure (Fig. 3) [25]. Moreover, precipitating factors of chronic heart failure are age-related, with a prevalence of infectious diseases and tachyarrhythmias in elderly against coronary attack and cardiac valve dysfunction in younger patients [26-28]. Furthermore, altered mental status (AMS) is common in elderly admitted to EDs with a ratio of $41-60 \%$ but the diagnosis is particularly difficult in these patients and it is also associated to higher mortality rates [29]. The results of a recent multicenter prospective study showed that infections (39.5\%) and neurological diseases (36.5\%) were the most common cause of AMS, followed by metabolic-electrolytic (17.3\%), cardiopulmonary (14.8\%), and gastrointestinal (5.7\%) diseases. Cardiac and pulmonary aetiologies were associated

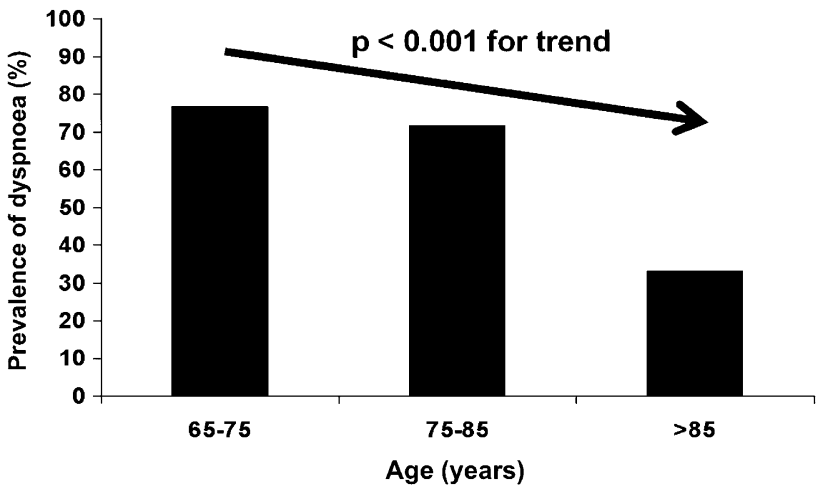

Fig. 3 Incidence of dyspnoea in elderly subjects with chronic heart failure. (with permission [25]) 
to $35 \%$ of AMS as showed by rates of delirium, AMS history, and mortality among etiologic groups.

Frequently, patients admitted in high intensity care for acute respiratory failure require NIV treatment, but despite strong evidences documenting NIV benefits, several studies have reported NIV failure rates ranging from 5 to $40 \%$. The possible causes of immediate failure (within min to $<1 \mathrm{~h}$ ) are a weak cough reflex, excessive secretions, hypercapnic encephalopathy, patient-ventilator asynchrony and psychomotor agitation due to patient intolerance [30]. In particular, elderly patients are at increased risk for the development of psychotic symptoms including agitation and uncooperative behaviour. In this scenario, the role of the Geriatrician becomes crucial because of its greater experience in the detection and management of psychotic behaviours which often worsen the already delicate condition of frail elderly patients [31]. Moreover, among the causes of NIV failure, delirium has been postulated to be an important contributing factor. In a prospective study investigating the rate of delirium in patients with acute respiratory failure treated by NIV and the potential association between delirium and mortality, among the 153 recruited subjects, more than $30 \%$ developed delirium and a strong association between delirium and subsequent mortality was found. Possible explanations of this relationship are the agitation, confusion and psychomotor disturbances associated to delirium, which reduce patient compliance (i.e. mask intolerance). These findings may explain why the 30-day survival is $95 \%$ in non-delirious group and $78 \%$ in the delirious group [32].

Therefore, carefully detection and management of these high-risk patients have a central role in reducing their mortality. For this purpose, in high intensity of care, the Geriatrician plays a decisive role in identifying and managing the atypical presentation of acute illness in elderly patients with comorbidity and related complications ("frail/complex" patients).

\section{Medium intensity}

A female, 93 years old, accesses to ED with acute abdominal pain. Her symptoms, physical examination, blood and imaging exams are showed in Table 2. Her medical history includes: hypertensive cardiomyopathy, hypercholesterolemia, gallstones and bilateral renal cysts. The patient is diagnosed with acute calculus cholecystitis. The therapy was performed as described in Table 2 . In addition, surgical intervention is indicated after the resolution of the acute condition. After few days, the general surgeon raises problems about patient surgery due to her old age. To quantify the surgical risk and the clinical frailty, the patient underwent a comprehensive geriatric assessment (CGA) by the consulted Geriatrician. After this evaluation, given the poor functional impairment and the low degree of clinical frailty, the patient underwent laparoscopic cholecystectomy under anaesthesia without complications.

"Ageism" and age discrimination are terms used in the literature to define negative attitudes towards older people. There is no reason for treating any individual badly, especially when they are particularly in need, because in this way we promote discrimination and failing the Hippocratic role of the healthcare professional which is "to cure sometimes, treat often and to comfort always". To avoid this phenomenon frailty units with well trained staff should be created, where frail older people can be comprehensively assessed and receive timely and targeted care, followed by a supported discharge [33]. In this sense, the CGA is particularly

Table 2 Medium intensity scenario

\begin{tabular}{|c|c|}
\hline \multicolumn{2}{|l|}{ Female, 93 years old } \\
\hline Symptoms & Right flank pain, nausea, vomiting, fever \\
\hline Vital signs & $\begin{array}{l}\text { BP } 140 / 85 \mathrm{mmHg} \\
\text { HR } 90 \text { beats } / \mathrm{min} \\
\text { RR } 21 \text { breaths } / \mathrm{min} \\
\text { T } 38.5^{\circ} \mathrm{C}\end{array}$ \\
\hline ECG & Sinus rhythm, right bundle branch block, left anterior hemiblock \\
\hline Abdominal X-ray & No air-fluid levels, no free gas in abdomen \\
\hline Abdominal ultrasonography & $\begin{array}{l}\text { Positive sonographic Murphy sign. Gallbladder wall thickening with a "binary" shape. Pericholecystic fluid. Intra- } \\
\text { and extra-hepatic biliary tract not dilated. Lithiasic formation at the level of the cystic duct }\end{array}$ \\
\hline Blood gas analysis & Within range \\
\hline Laboratory tests & $\begin{array}{l}\text { WBC 16,500 mm } \text { m }^{3} \text { (neutrophils } 90.4 \% \text { ), fibrinogen } 560 \mathrm{mg} \% \text {, glycemia } 320 \mathrm{mg} \% \text {, direct bilirubin } 2.2 \mathrm{mg} \% \text {, GGT } \\
52 \mathrm{U} / \mathrm{L} \text {, ALP } 224 \mathrm{U} / \mathrm{L} \text {, AST } 52 \mathrm{U} / \mathrm{L} \text {, ALT } 74 \mathrm{U} / \mathrm{L}\end{array}$ \\
\hline Therapy & $\begin{array}{l}\text { Piperacillin-tazobactam } 4.5 \mathrm{~g} \text { e.v./8 h, metoclopramide } 1 \mathrm{fl} \text { e.v./8 h, ketorolac } 30 \mathrm{mg} \text { e.v./8 h, omeprazole } 20 \mathrm{mg} \\
\text { e.v./1 h }\end{array}$ \\
\hline
\end{tabular}

$B P$ blood pressure, $H R$ heart rate, $b p m$ beats per minute, $R R$ respiratory rate, $T$ body temperature, $E C G$ electrocardiogram, $W B C$ white blood cells 
useful because it explores the functional, nutritional and socio-economic status, comorbidity and emotional and cognitive functions, providing a global assessment of the elderly patient. The evaluation of the surgical risk together with different prognostic surgical validated tools represents the best approach to this kind of patient. A recent study showed that the use of some simple indices of CGA, together with surgical prognostic indices (the physiological and operative severity score for the enumeration of mortality and morbidity-Possum, the surgical risk score-SRS defined by the National Confidential Enquiry into Patient Outcome of Death, the American Society of Anaesthesiologists-ASA, and the British United Provident Association-BUPA) can provide valuable help in identifying the highest risk patients to develop negative outcomes after surgery. In particular, multivariate analysis showed that higher cognitive function assessed by MMSE is associated with a reduced risk of total mortality, while comorbidity is associated with a higher risk of total morbidity [34].

Therefore, in medium intensity of care, the Geriatrician is frequently involved in the administration of CGA and identification and quantification of clinical frailty because some medical procedures are not performed only for the advanced age of the patients.

\section{Low intensity}

A 78-year-old male, widower, accesses to the ED for an apparently accidental fall. He is led to the hospital after the intervention of the firefighters warned by a neighbour. The patient was found on the ground, awake, with no signs of trauma, unable to rise, confused about time and space and he did not remember what was happened. His physical examination, blood and imaging exams are showed in Table 3. His medical history includes arterial hypertension in pharmacological treatment, prostatic adenocarcinoma treated with radical prostatectomy, moderate chronic kidney disease and hypercholesterolemia. Due to the unclear dynamics of the fall, a cardiologic and neurologic counselling is required, showed in Table 3. Finally, a geriatric counselling is required. During orthostatic blood pressure (BP) measurements, in recumbency position is recorded a BP of $110 / 75 \mathrm{mmHg}$, but at the first minute of orthostatism, BP of $75 / 60 \mathrm{mmHg}$ was recorded, thus a diagnosis of iatrogenic orthostatic syncope was performed. Since the iatrogenic nature of the syncope, the cognitive decline associated with disability and the presence of a precarious socio-environmental state, the Geriatrician plans a "protected" discharge in a nursing home.

Table 3 Low intensity scenario

\begin{tabular}{|c|c|}
\hline \multicolumn{2}{|l|}{ Male, 78 years old } \\
\hline Vital signs & $\begin{array}{l}\text { BP } 110 / 70 \mathrm{mmHg} \\
\text { HR } 55 \text { beats/min } \\
\text { RR } 18 \text { breaths/min }\end{array}$ \\
\hline ECG & Sinus tachycardia \\
\hline Physical exam & Negative \\
\hline Chest X-ray & VII and VIII left ribs infractions \\
\hline Abdominal ultrasonography & Negative \\
\hline Head CT & No haemorrhagic parenchymal lesions. Cortical and subcortical parenchymal atrophy \\
\hline Cardiologic counselling & $\begin{array}{l}\text { Cardiac exam: negative } \\
\text { ECG: sinus bradycardia } \\
\text { Echocardiography: left ventricular hypertrophy, diastolic dysfunction, EF: } 55 \% \\
\text { Required } 24 \text { h ECG Holter }\end{array}$ \\
\hline Neurologic counselling & $\begin{array}{l}\text { EEG: negative } \\
\text { Supraaortic vessels examination: No hemodynamically significant stenosis } \\
\text { Mild cognitive deficit } \\
\text { Required control head CT }\end{array}$ \\
\hline Geriatric counselling & $\begin{array}{l}\text { CGA } \\
\text { Pharmacological therapy } \\
\text { Ramipril } 5 \mathrm{mg} 1 \mathrm{cp} \\
\text { Dexazosin } 4 \mathrm{mg} 1 \mathrm{cp} \\
\text { Bisoprolol-Idroclorotiazide } 5-12.5 \mathrm{mg} 1 \mathrm{cp} \\
\text { Acetylsalicylic acid } 100 \mathrm{mg} 1 \mathrm{cp} \\
\text { Atorvastatin } 20 \mathrm{mg} 1 \mathrm{cp} \mathrm{la} \mathrm{sera} \\
\text { BADL } 4 / 6 \text { (lost functions), IADL } 8 / 8 \text { (lost functions) } \\
\text { MMSE } 19 / 30\end{array}$ \\
\hline
\end{tabular}

$B P$ blood pressure, $H R$ heart rate, $b p m$ beats per minute, $R R$ respiratory rate, $E C G$ electrocardiogram, $E F$ ejection fraction, $E E G$ electroencephalogram, $C T$ computed tomography, $C G A$ comprehensive geriatric assessment, $B A D L$ and $I A D L$ basic and instrumental activity of daily living, MMSE mini-mental state examination 
Falls are a leading cause of injury and death among older adults and a significant public health issue [35]. There are syncopal and non-syncopal causes of fall in older people. The main causes of syncopal falls in older adults are: (1) orthostatic hypotension, (2) carotid sinus syndrome, (3) various forms of neutrally mediated syncope and (4) cardiac arrhythmias. Causes of non-syncopal falls include (1) disorders without any impairment of consciousness (cataplexy, drop attacks, psychogenic pseudo-syncope, transient ischaemic attacks ITIA of carotid origin) and (2) disorders with any impairment of consciousness (metabolic disorders as hypoglycaemia, hypoxia, hyperventilation with hypocapnia, Epilepsy, intoxications, vertebro-basilar TIA) [36].

The Prevention of Falls Network Europe (ProFANE) group defined a fall as "an unexpected event in which the participant comes to rest on the ground, floor, or lower level." [37]. Fall risk factors are often divided in intrinsic and extrinsic. Intrinsic risk factors for falls can be divided in: demographic (age, gender, race), systemic (gait and balance, strength, vision and cognition) and symptoms/diseases (dizziness/vertigo, cardiovascular disease, dementia, depression). Extrinsic risk factors for falls include drugs (psychotropic medications, such as benzodiazepines, diabetes medications, non-steroidal anti-inflammatory drugs, cardiovascular medications such as digoxin, type 1a antiarrhythmic and diuretics, antiepileptics), home environment (poor lighting or objects around the home) and footwear (slippers, walking barefoot, socks increase the risk of falls compared to walking with athletic or canvas shoes) [38].

Among the syncopal causes of falls, orthostatic hypotension is one of the leading causes in the elderly and can be easily recognized by physical examination. However, its prevalence is difficult to assess, because the relation between hypotension and a syncopal episode is sometimes difficult to establish and syncope may have a multifactorial aetiology. A study assessed the prevalence of syncope due to orthostatic hypotension in the ED and described the clinical characteristics of these patients: patients with orthostatic hypotension were significantly older had more comorbid conditions, and the number of prescribed hypotensive medications was higher $(\geq 2)$ [39]. Recently, the Syncope and Dementia (SYD) study, a prospective, observational, multicentre study conducted to define the aetiology of syncope and unexplained falls in elderly adults with dementia, showed that the most frequent cause of syncope and dementia in this elderly population was orthostatic hypotension $(\approx 50 \%)$ [33]. Hypertension is one of the most frequent comorbidity in geriatric population and it is frequently associated to cognitive decline [40]. Elderly patients with hypertension and cognitive decline show a progressive reduction of blood pressure values, probably related to a "vascular dysautonomy", in turn related to chronic cerebral hypoperfusion [40, 41].
Therefore, frail elderly people may have the necessity to be managed through an integrated network of services. Elderly patients should undergo a CGA by the Geriatric Evaluation Unit, consisting of various healthcare professionals, such as general practitioners (GPs), professional nurses, social workers and Geriatricians. Through the CGA, it is possible to establish elderly patients' needs of care and then address them to the service that best suits their needs. Hospital facilities are destined to major acute diseases which require diagnostic interventions of a certain technological complexity or particularly complex therapies. Territorial services include integrated home care, hospital at home care, daytime care centres and healthcare homes. All these services are born to ensure the patients continuity of care at the moment of discharge (Fig. 4). Therefore, in low intensity of care, the Geriatrician is involved in ensuring continuity of care by directing the patient to the structure of the geriatric services network most appropriate to his case. Despite the importance of these structures, the percentage of nursing homes and integrated home cares is poor in Italy, especially in Regione Campania (Abete, unpublished data). Given the increase of the elderly population, it is evident that the integrated network of geriatric territorial services is inadequate to meet the needs of this complex and demanding population. There are several difficulties in creating a geriatric services network. First of all, a change of culture is required, at both clinical and management levels, to avoid problems and a lack of shared vision in the long-term sustainability of integration services. Many health systems have pre-existing divisions in financing, management structure, and care provision, which preclude the implementation of integrated care. Regulation is exerted over specific providers such as hospitals and community health services, while in an integrated care, model regulation must shift to be exerted on services across a continuum of care or a care package. Integrated network services require carefully developed partnerships, finance structures, and information technology platforms to provide adequately protected support and funding [42].

\section{Potential advantages/disadvantages between intensity of care model and traditional model}

The potential advantages and disadvantages between intensity of care model and traditional model are shown in Table 4. In particular, the "intensity of care" model is characterized by improvement of assistance and care, which is "patient-centred", optimization of human and economic resources and enhancement of collaboration between different specialists. However, the main disadvantage is the 
Fig. 4 An example of integrated network of geriatric territorial services

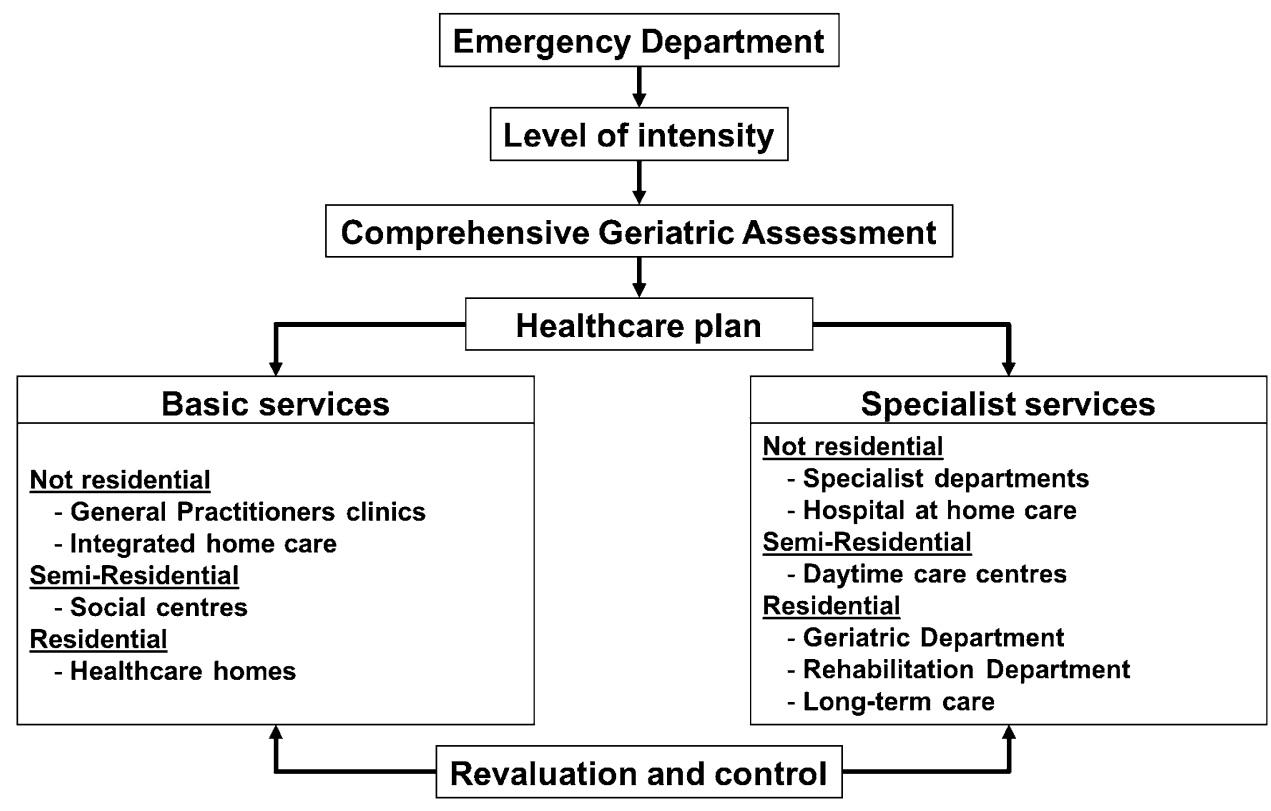

Table 4 Advantages and disadvantages of "intensity of care" model and "traditional model"

\begin{tabular}{|c|c|}
\hline Intensity of care model & Traditional care model \\
\hline \multicolumn{2}{|l|}{ Advantages } \\
\hline Improvement of care and assistance & More sophisticated specialized knowledge and operating techniques \\
\hline Improvement in patient care and comfort & Constant attention to improving the cost/benefit ratio \\
\hline Optimization of human and economic resources & $\begin{array}{l}\text { Easier management because patients are admitted in specialist ward accord- } \\
\text { ing to their main pathology }\end{array}$ \\
\hline Improvement of collaboration between different specialists & Division of tasks for specializations and hierarchical levels \\
\hline \multicolumn{2}{|l|}{ Disadvantages } \\
\hline $\begin{array}{l}\text { Need for economic and human resources for the reorganization } \\
\text { of hospital facilities }\end{array}$ & Assistance and care disease-centered \\
\hline Possible conflicts among different healthcare professionals & The healthcare professional worker is the only active part of the care process \\
\hline Difficulty in accepting the new organizational model & Exclusion of the patient and his caregivers from treatment choices \\
\hline
\end{tabular}

cultural difficulty in accepting this new type of organization, which requires high economic and human resources.

In addition, to address the complex needs of frail hospitalized older persons, two different approaches have been postulated. The first is the "CGA team model", in which frail older patients are hospitalized on a non-geriatric wardbased on the patient's main medical reason for admission-and evaluated by a multidisciplinary team referred to as the inpatient geriatric consultation team (IGCT) which recommend a plan of treatment. However, a meta-analysis about the impact of geriatric consultation teams on clinical outcome in acute hospitals, including 20 studies evaluating 4,546 participants, observed only a beneficial effect on mortality at 6 and 8 months post-hospital discharge, without an effect on functional status, length of stay and readmission rate [43]. The alternative approach is the geriatric co-management, which is characterized by the shared responsibility and decision making between a physician (e.g. surgeon) and a Geriatrician, who provides complementary medical care in the prevention and management of geriatricoriented problems [44]. Co-management interventions have previously been evaluated in systematic reviews focusing on orthogeriatric care showing a significant reduction of inhospital and long-term mortality and length of stay [45]. A recent meta-analysis about the effectiveness of in-hospital geriatric co-management, including 20 studies and 3,590 patients, showed low-quality evidence of a reduced length of stay and a reduced number of patients with complications, and very low-quality evidence of better functional status as a result of geriatric co-management [44].

Theoretically, the CGA team model fits better with the "traditional model" of care, because the elderly patient is admitted to a specialist ward and the Geriatrician is contacted only in case of need to guide the therapeutic choices. 
In contrast, in "intensity of care" model, the geriatric comanagement system seems to be the most suitable: different specialists, including the Geriatrician, working together to choose the best therapeutic approach aimed at satisfying the needs of care of the whole patient and not only according to his/her basic specific pathology.

\section{Conclusions and future perspectives}

The intensity of care model is based on a clinical approach centred on the person and his needs of care and not only on his pathologies. The transition to this health system from the traditional one offers a greater human and economic resources optimization.

Geriatrician is the professional figure that best suits this model of care, since the complexity and frailty of elderly patients always requires a multidisciplinary approach to patient care. In high intensity of care, the Geriatrician plays a key role in identifying the atypical presentation of acute illness in elderly patients with comorbidity and the prevention and management of related complications. In medium intensity of care, it is involved in the administration of CGA and identification and quantification of clinical frailty, to improve the management of hospitalized frail elderly patients, while in low intensity of care the Geriatrician provides continuity of care through the activation of the territorial network of services more appropriate to the patient needs.

The emerging demand, by the National Health System, of a multidisciplinary approach to patient care, typical of Geriatricians, leads to what Tinetti calls "geriatric paradox": the increasing interest in small "g" geriatrics (the geriatric multidimensional approach to patients care) in contrast with the few interest in big "G" Geriatrics (the training and support of the field of Geriatrics or the expertise of Geriatricians) [46]. The greater risk is the "theft" of the geriatric approach to the patient and the consequent "extinction" of the figure of the Geriatrician. The only way to allow integration, rather than exclusion, of geriatrics in clinical practice is letting the world know, clearly and positively, who we are and what we do.

\section{Compliance with ethical standards}

Conflict of interest The authors declare that they have no conflict of interest.

Statement of human and animal rights All procedures performed in studies involving human participants were in accordance with the ethical standards of the institutional and/or national research committee and with the 1964 Helsinki declaration and its later amendments or comparable ethical standards.
Informed consent Informed consent was obtained from all individual participants included in the study.

\section{References}

1. Artioli G, Foà C, Taffurelli C (2016) An integrated narrative nursing model: towards a new healthcare paradigm. Acta Bio Med Atenei Parm 87:13-22

2. Bardes CL (2012) Defining "patient-centered medicine". N Engl J Med 366:782-783. https://doi.org/10.1056/NEJMp1200070

3. Engel GL (2012) The need for a new medical model: a challenge for biomedicine. Psychodyn Psychiatry 40:377-396. https://doi. org/10.1521/pdps.2012.40.3.377

4. Chesi G, Nardi R (2013) Severity stratification of patients hospitalized on the internal medicine ward: work in progress. Ital J Med 7:231-233. https://doi.org/10.4081/itjm.2013.231

5. NardI R, Arienti V, Nozzoli C et al (2012) Hospital organization based on intensity of care: potential errors to avoid. Ital J Med 6:1-13. https://doi.org/10.4081/itjm.2012.1

6. Roberts DC, McKay MP, Shaffer A (2008) Increasing rates of emergency department visits for elderly patients in the United States, 1993-2003. Ann Emerg Med 51:769-774. https://doi. org/10.1016/j.annemergmed.2007.09.011

7. Galizia G, Cacciatore F, Testa G et al (2011) Role of clinical frailty on long-term mortality of elderly subjects with and without chronic obstructive pulmonary disease. Aging Clin Exp Res 23:118-125

8. Cacciatore F, Testa G, Galizia G et al (2013) Clinical frailty and long-term mortality in elderly subjects with diabetes. Acta Diabetol 50:251-260. https://doi.org/10.1007/s00592-012-0413-2

9. Hastings SN, Schmader KE, Sloane RJ et al (2007) Adverse health outcomes after discharge from the emergency department-incidence and risk factors in a veteran population. J Gen Intern Med 22:1527-1531. https://doi.org/10.1007/s11606-007-0343-9

10. McCusker J, Cardin S, Bellavance F et al (2000) Return to the emergency department among elders: patterns and predictors. Acad Emerg Med Off J Soc Acad Emerg Med 7:249-259

11. Limpawattana P, Phungoen P, Mitsungnern T et al (2016) Atypical presentations of older adults at the emergency department and associated factors. Arch Gerontol Geriatr 62:97-102. https://doi. org/10.1016/j.archger.2015.08.016

12. Romero-Ortuno R, Wallis S, Biram R et al (2016) Clinical frailty adds to acute illness severity in predicting mortality in hospitalized older adults: an observational study. Eur J Intern Med 35:24-34. https://doi.org/10.1016/j.ejim.2016.08.033

13. Di Bari M, Balzi D, Roberts AT et al (2010) Prognostic stratification of older persons based on simple administrative data: development and validation of the "Silver Code," to be used in emergency department triage. J Gerontol A Biol Sci Med Sci 65:159-164. https://doi.org/10.1093/gerona/glp043

14. Haldeman JC (1959) Progressive patient care: a challenge to hospitals and health agencies. Public Health Rep Wash DC 1896 74:405-408

15. De Pietro C, Benvenuti C, Sartirana M (2011) Gli ospedali per intensità di cura in Toscana: un'esperienza in corso. L'aziendalizzazione della sanità in Italia. Rapporto Oasi 2011, Milano

16. Nuovi modelli di organizzazioni ospedaliere: l'esperienza vincente del week hospital dell'azienda ospedaliera città della salute e della scienza di Torino-P.O. CTO-Maria Adelaide-Marzia Bonfanti, Emanuele Porazzi, Gianluca Collo, Maria Adele Schirru—autori-vari-Maggioli Editore-Articolo Digital Magazine Maggioli 6:79-91 
17. Croce D. Le innovazioni organizzative in ambito ospedaliero: $i$ meccanismi operativi del S. Gerardo diMonza Sanità Pubblica e Privata N. 4/2007 Maggioli editore Rimini

18. Moroni P, Colanghi E, Bonfanti M (2011) Nuovi "modelli modulari di cura": l'intensità di cura a dimensione variabile. Il caso dell'Azienda Ospedaliera di Desio e Vimercate. Sanit Pubbl E Priv 3:1-72

19. Zoppini L, Lombardo M, Cordone A et al (2010) L'organizzazione dell'ospedale moderno. Studio di riorganizzazione dell'attività chirurgica dell'A.O. Ospedale civile di Legnano secondo il modello hub \& spoke e per intensità di cura. Organ Sanit XXXIV:5-34

20. Nicosia F (2008) Dossier Ospedale "lean" per intensità di cureOspedali Galliera. Manag Della Sanit 12:36-40

21. Orlandi W, Duca E, Pioppo M (2006) L'ospedale per aree di intensità di cura omogenee e di assistenza multi specialistica: l'esperienza dell'Azienda USL n. 3 dell'Umbria. Organ Sanit 4:35-40

22. Alesani D, Barbieri M, Lega F et al (2006) Gli impatti delle innovazioni dei modelli logistico-organizzativi in ospedale: spunti da tre esperienze aziendali pilota. Rapporto OASI 2006. Egea, Milano

23. Sebastiano A, Croce D (2007) Linee evolutive nello sviluppo organizzativo delle strutture ospedaliere: case history e riflessioni sistemiche 6:34-38

24. Guarinoni MG, Motta PC, Petrucci C et al (2013) Progressive patient care model and its application into hospital organization: a narrative review. Prof Inferm 66:205-214

25. Cacciatore F, Gallo C, Ferrara N et al (1998) Morbidity patterns in aged population in southern Italy. A survey sampling. Arch Gerontol Geriatr 26:201-213

26. Abete $\mathrm{P}$, Cacciatore F, Ferrara N et al (2003) Body mass index and preinfarction angina in elderly patients with acute myocardial infarction. Am J Clin Nutr 78:796-801

27. Testa G, Della-Morte D, Cacciatore F et al (2013) Precipitating factors in younger and older adults with decompensated chronic heart failure: are they different? J Am Geriatr Soc 61:1827-1828. https://doi.org/10.1111/jgs.12475

28. Cacciatore F, Testa G, Langellotto A et al (2012) Role of ventricular rate response on dementia in cognitively impaired elderly subjects with atrial fibrillation: a 10-year study. Dement Geriatr Cogn Disord 34:143-148. https://doi.org/10.1159/000342195

29. Xiao H-Y, Wang Y-X, Xu T et al (2012) Evaluation and treatment of altered mental status patients in the emergency department: life in the fast lane. World J Emerg Med 3:270-277. https://doi. org/10.5847/wjem.j.1920-8642.2012.04.006

30. Ozyilmaz E, Ugurlu AO, Nava S (2014) Timing of noninvasive ventilation failure: causes, risk factors, and potential remedies. BMC Pulm Med 14:19. https://doi.org/10.1186/1471-2466-14-19

31. Chan W, Lam LC, Chen EY (2011) Recent advances in pharmacological treatment of psychosis in late life. Curr Opin Psychiatry 24:455-460. https://doi.org/10.1097/YCO.0b013e32834a3f47

32. Chan K-Y, Cheng LSL, Mak IWC et al (2017) Delirium is a strong predictor of mortality in patients receiving non-invasive positive pressure ventilation. Lung 195:115-125. https://doi.org/10.1007/ s00408-016-9955-3

33. Ungar A, Mussi C, Ceccofiglio A et al (2016) Etiology of syncope and unexplained falls in elderly adults with dementia: syncope and dementia (SYD) study. J Am Geriatr Soc 64:1567-1573. https:// doi.org/10.1111/jgs.14225

34. Abete P, Cherubini A, Di Bari M et al (2016) Does comprehensive geriatric assessment improve the estimate of surgical risk in elderly patients? An Italian multicenter observational study. Am J Surg 211:76-83.e2. https://doi.org/10.1016/j.amjsurg.2015.04.016

35. Tinetti ME (2003) Clinical practice. Preventing falls in elderly persons. N Engl J Med 348:42-49. https://doi.org/10.1056/NEJMc p020719

36. Brignole M, Alboni P, Benditt DG et al (2004) Guidelines on management (diagnosis and treatment) of syncope-update 2004. Exec Summ Eur Heart J 25:2054-2072. https://doi.org/10.1016/j. ehj.2004.09.004

37. Lamb SE, Jørstad-Stein EC, Hauer K et al (2005) Development of a common outcome data set for fall injury prevention trials: the Prevention of Falls Network Europe consensus. J Am Geriatr Soc 53:1618-1622. https://doi.org/10.1111/j.1532-5415.2005.53455 . $\mathrm{x}$

38. Ambrose AF, Paul G, Hausdorff JM (2013) Risk factors for falls among older adults: a review of the literature. Maturitas 75:51-61. https://doi.org/10.1016/j.maturitas.2013.02.009

39. Sarasin FP, Louis-Simonet M, Carballo D et al (2002) Prevalence of orthostatic hypotension among patients presenting with syncope in the ED. Am J Emerg Med 20:497-501

40. Cacciatore F, Abete P, de Santis D et al (2005) Mortality and blood pressure in elderly people with and without cognitive impairment. Gerontology 51:53-61. https://doi.org/10.1159/000081436

41. Abete P, Della-Morte D, Gargiulo G et al (2014) Cognitive impairment and cardiovascular diseases in the elderly. A heart-brain continuum hypothesis. Ageing Res Rev 18:41-52. https://doi. org/10.1016/j.arr.2014.07.003

42. Maruthappu M, Hasan A, Zeltner T (2015) Enablers and barriers in implementing integrated care. Health Syst Reform 1:250-256. https://doi.org/10.1080/23288604.2015.1077301

43. Deschodt M, Flamaing J, Haentjens P et al (2013) Impact of geriatric consultation teams on clinical outcome in acute hospitals: a systematic review and meta-analysis. BMC Med 11:48. https:// doi.org/10.1186/1741-7015-11-48

44. Van Grootven B, Flamaing J, Dierckx de Casterlé B et al (2017) Effectiveness of in-hospital geriatric co-management: a systematic review and meta-analysis. Age Ageing 46:903-910. https://doi. org/10.1093/ageing/afx051

45. Grigoryan KV, Javedan H, Rudolph JL (2014) Orthogeriatric care models and outcomes in hip fracture patients: a systematic review and meta-analysis. J Orthop Trauma 28:e49-55. https:// doi.org/10.1097/BOT.0b013e3182a5a045

46. Tinetti M (2016) Mainstream or extinction: can defining who we are save geriatrics? J Am Geriatr Soc 64:1400-1404. https://doi. org/10.1111/jgs. 14181 